\title{
Non-toxic phototriggered gene transfection by PAMAM-porphyrin conjugates
}

\author{
Ming-Jium Shieh ${ }^{\mathrm{a}, \mathrm{b}}$, Cheng-Liang Peng ${ }^{\mathrm{a}}$, Pei-Jen Lou ${ }^{\mathrm{c}}$, Chieh-Hua Chiu ${ }^{\text {a }}$, Tsiao-Yu Tsai ${ }^{\mathrm{d}}$, \\ Chia-Yen Hsu ${ }^{\mathrm{d}}$, Chen-Yu Yeh ${ }^{\mathrm{d}}$, Ping-Shan Lai ${ }^{\mathrm{d}, *}$ \\ a Institute of Biomedical Engineering, College of Medicine and College of Engineering, National Taiwan University, No. 1, Section 1, Jen-Ai Road, Taipei 100, Taiwan \\ b Department of Oncology, National Taiwan University Hospital and College of Medicine, No. 7, Chung-Shan South Road, Taipei 100, Taiwan \\ c Department of Otolaryngology, National Taiwan University Hospital and College of Medicine, No. 7, Chung-Shan South Road, Taipei 100, Taiwan \\ d Department of Chemistry, National Chung Hsing University, No. 250, Kuo-Kuang Road, Taichung 402, Taiwan
}

\section{A R T I C L E I N F O}

\section{Article history:}

Received 7 January 2008

Accepted 31 March 2008

Available online 25 April 2008

\section{Keywords:}

Polyamidoamine dendrimer

Photochemical internalization

Cytotoxicity

Transfection

\begin{abstract}
A B S T R A C T
Development of controllable and non-toxic gene transfection systems is a core issue in gene therapy. Photochemical internalization, an innovative strategy in cytosolic release, provides us with an opportunity to develop a light-inducible gene delivery system. In this study, a novel photochemical internalization (PCI)mediated gene delivery system was synthesized by surface modification of polyamidoamine (PAMAM) dendrimers via 5,10,15-tri(4-acetamidophenyl)-20-mono(4-carboxyl-phenyl)porphyrin (TAMCPP) conjugated to the generation 4 PAMAM dendrimer (G4). This water-soluble PAMAM-TAMCPP conjugate was characterized for cell viability, phototoxicity, DNA complexation, and in vitro transfection activity. The results show that TAMCPP conjugation did not increase the cytotoxicity of the PAMAM dendrimer below $20 \mu \mathrm{M}$, but significantly induced cell death after suitable irradiation. Under almost non-toxic G4-TAMCPP-mediated PCI treatment, the expression of green fluorescent protein determined by flow cytometry could be markedly enhanced in HeLa cells. Therefore, the G4-TAMCPP conjugate had an inducible and effective gene transfection activity, and showed considerable potential as a bimodal biomaterial for $\mathrm{PCI}$-mediated gene therapy.
\end{abstract}

Crown Copyright @ 2008 Published by Elsevier B.V. All rights reserved.

\section{Introduction}

Gene therapy is a promising strategy to deliver desired gene into target cells for the treatment of genetic deficiencies. Viral vectors have been applied in gene delivery because of their high transfection efficacy. However, their safety concerns, including mutagenesis and immunogenicity, affect their broad application in the clinic $[1,2]$. Non-viral carriers, such as cationic polymer, have been developed as an alternative delivery strategy with less immunogenicity and lower cost $[3,4]$. Among these non-viral vectors, polyamidoamine (PAMAM) dendrimer, a novel and unique synthetic macromolecule with a 3-dimensional highly branched structure, is widely used in gene delivery [5,6]. The capability of PAMAM dendrimers to transfect cells appears to depend on the generation and number of primary amino groups on the surface of the polymer. However, generation-dependent cytotoxicity of PAMAM dendrimers has been shown in previous studies [7,8]. Thus, the development of PAMAM dendrimers with low generation, low toxicity, and high transfection efficiency is an important area of research in dendrimer-based gene delivery.

Many studies have demonstrated that surface modification by hydrophobic moieties, such as fluorescent dyes and amino acids, affect

\footnotetext{
* Corresponding author. Tel.: +886 4 22840411x409; fax: +886 422367269

E-mail address: f89548003@gmail.com (P.-S. Lai).
}

the oligonucleotide or gene transfection activity of PAMAM dendrimers $[9,10]$. Although an enhanced activity can be observed with these systems, the transfection process cannot be controlled by a designed switch. Conjugating an appropriate amount of functionalized hydrophobic molecules to the PAMAM dendrimer to trigger the transfection process and elevate its transfection activity may provide a powerful tool for gene therapy.

Photodynamic therapy (PDT) is a photochemical process for producing localized tissue necrosis, which involves the activation of a photosensitizing drug in the target tissue with light of a specific wavelength matched to an absorption peak of the photosensitiser in the presence of molecular oxygen [11]. It is a recognized therapeutic modality, which has regulatory approval for the treatment of a variety of human pre-malignant and malignant diseases. Photochemical internalization (PCI), a specific branch of PDT, is a novel technology utilized for the site-specific release of macromolecules within cells. The mechanism of $\mathrm{PCI}$ is based on the breakdown of the endosomal/ lysosomal membranes by photoactivation of photosensitizers that localize on the membranes of these organelles [12]. The PCI strategy has been utilized to release macromolecules such as toxins, DNA delivered as a complex with cationic polymers or incorporated in adenovirus or adeno-associated virus, dendrimer-doxorubicin conjugates, peptide nucleic acids, and bleomycin, from endocytic vesicles to the cytosol [13-19]. Furthermore, PCI can reverse the adriamycinresistance of breast cancer cells [20]. 
To develop an inducible and low toxic vector, we used a generation 4 PAMAM dendrimer as a gene carrier, and implemented the $\mathrm{PCI}$ strategy by conjugating a hydrophobic porphyrin to facilitate its cytosolic release and improve the transfection activity. The cytotoxicity, phototoxicity, and efficacy of the resulting complex were evaluated using HeLa cells as a model, and using the MTT assay and transfection experiments. Our results show that the gene transfection activity of the PAMAM-porphyrin conjugate can be controlled by irradiation under non-toxic conditions, and can be considered as a novel PCI-mediated gene delivery system.

\section{Materials and methods}

\subsection{Materials}

Generation 4 polyamidoamine (PAMAM) dendrimer was purchased from Aldrich Chemical Company, Inc. (Milwaukee, WI) and all other chemicals were from Sigma Chemical Co. (St. Louis, MO).

\subsection{Synthesis of meso-substituted porphyrins and PAMAM-porphyrin conjugate}

5,10,15-tri(4-acetamidophenyl)-20-mono(4-carboxyl-phenyl)porphyrin (TAMCPP) was synthesized from a binary mixture of aldehydes and pyrrole using a modification of the Alder-Longo method as showed in Scheme 1 [21]. ${ }^{1} \mathrm{H}$ NMR (400 MHz, D6-DMSO): $\delta$ (ppm) 8.86 (pyrrole, $s, 6 \mathrm{H}$ ); 8.81 (pyrrole, $s, 2 \mathrm{H}$ ); 8.38 (benzoic acid, $d, J=6.8 \mathrm{~Hz}$, $2 \mathrm{H}$ ); 8.23 (benzoic acid, $d, J=8.4 \mathrm{~Hz}, 2 \mathrm{H}$ ); 8.12 (aniline, $d, J=7.6 \mathrm{~Hz}$, $6 \mathrm{H}$ ); 8.04 (aniline, $d, J=7.6 \mathrm{~Hz}, 6 \mathrm{H}) ; 2.21\left(\mathrm{NH}(\mathrm{C}=\mathrm{O}) \mathrm{CH}_{3}, s, 9 \mathrm{H}\right)$.

In order to conjugate TAMCPP to the PAMAM dendrimer, TAMCPP was first activated by dicyclohexylcarbodiimide (DCC) and $\mathrm{N}$-hydroxysuccinimide (NHS) and yielded the desired TAMCPP-
NHS. PAMAM dendrimer-TAMCPP conjugates (G4-TAMCPP) were prepared by reacting G4 PAMAM dendrimers with TAMCPP-NHS. After $48 \mathrm{~h}$ at room temperature, the reaction mixtures were dialyzed for another $48 \mathrm{~h}$ using a molecular weight cut-off (MWCO) of 10,000 membranes successively against hydrochloric acid/aqueous/methanol solution (0.5/49.5/50). The red conjugate solutions were further concentrated under reduced pressure at $35{ }^{\circ} \mathrm{C}$ and the resulting solutions were frozen in liquid nitrogen and lyophilized to obtain the conjugate.

The conjugate was characterized using thin layer chromatography (TLC) and the degree of porphyrin conjugated to PAMAM dendrimer was estimated as $12 \%(\mathrm{w} / \mathrm{w})$ by a Cary 50 spectrophotometer (Varian Inc., Palo Alto, CA, USA) using the soret molar extinction coefficients of TAMCPP (log $\sim 5.62$ at $420 \mathrm{~nm}$ ). The lipophilic and hydrophilic properties of G4-TAMCPP were characterized by the partition coefficient $P_{\mathrm{o} / \mathrm{w}}=C_{\mathrm{o}} / C_{\mathrm{w}}$ of the compound between the two immiscible solvents $n$-octanol (o) and water (w) [22]. The morphology of G4TAMCPP was observed by transmission electron microscopy (TEM) (Hitachi H-7500) operating at $120 \mathrm{kV}$.

\subsection{Cell lines and culture conditions}

Human cervical epithelioid carcinoma (HeLa) cells were maintained in a humidified $5 \% \mathrm{CO}_{2}$ incubator at $37^{\circ} \mathrm{C}$ in DMEM (Gibco BRL, Gaithersburg, MD) supplemented with $10 \%$ heat-activated fetal bovine serum (FBS) (Gibco BRL, Gaithersburg, MD) and 1\% antibiotics (Antibiotic-Antimycotic, Gibco BRL, Gaithersburg, MD, USA).

\subsection{Cellular uptake of photosensitizing agents}

Cellular uptake of TAMCPP or G4-TAMCPP was measured by fluorometry. Cells $\left(1.5 \times 10^{5}\right)$ were incubated with one of the

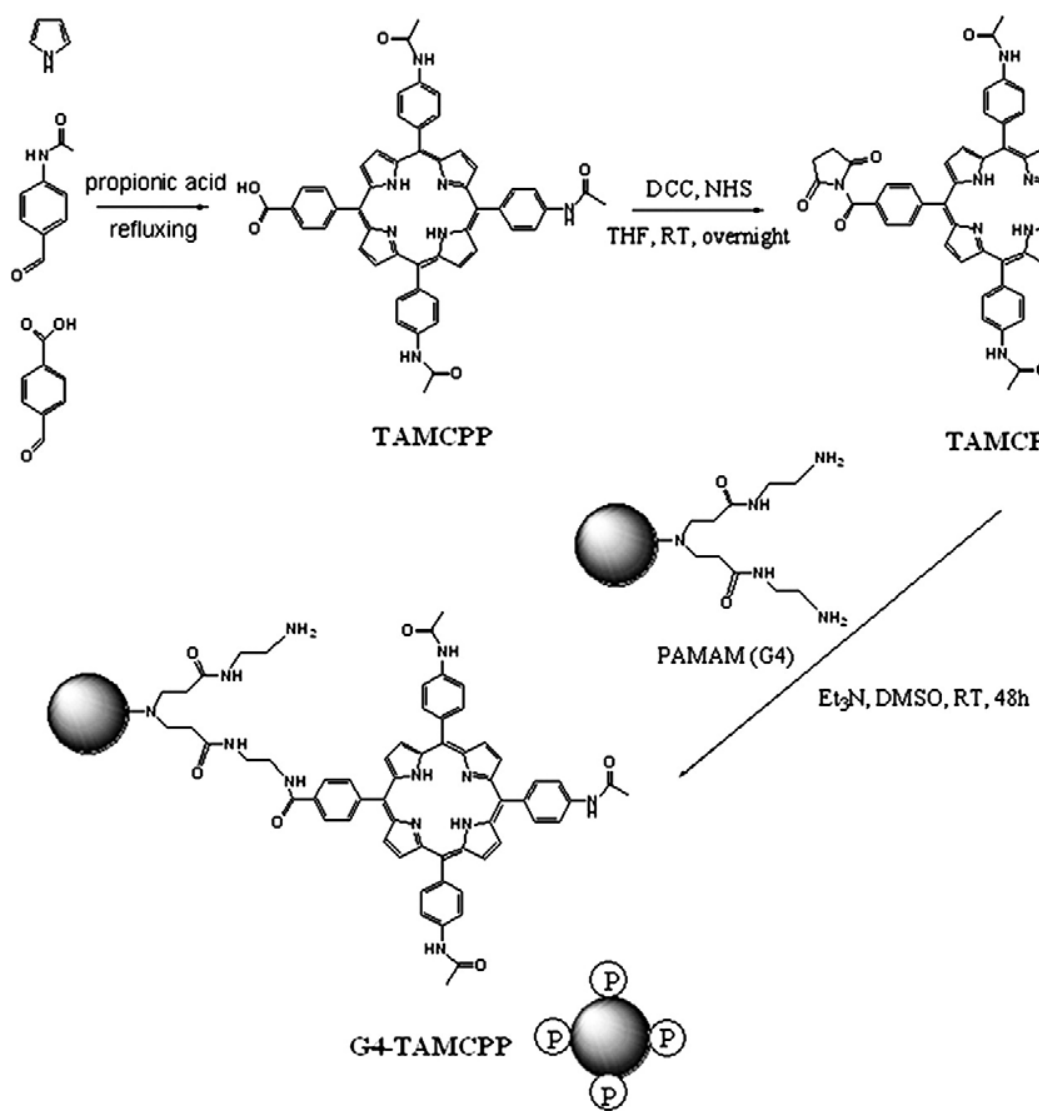

Scheme 1. Schematic representation of TAMCPP and G4-TAMCPP conjugate synthesis. 


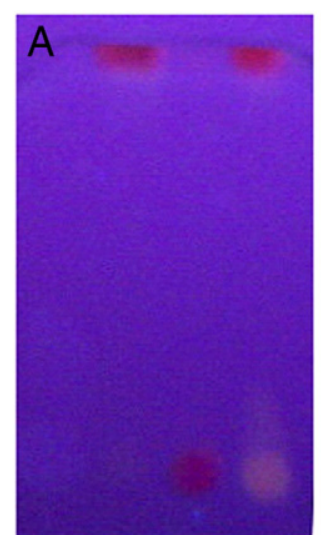

$\begin{array}{llll}1 & 2 & 3 & 4\end{array}$

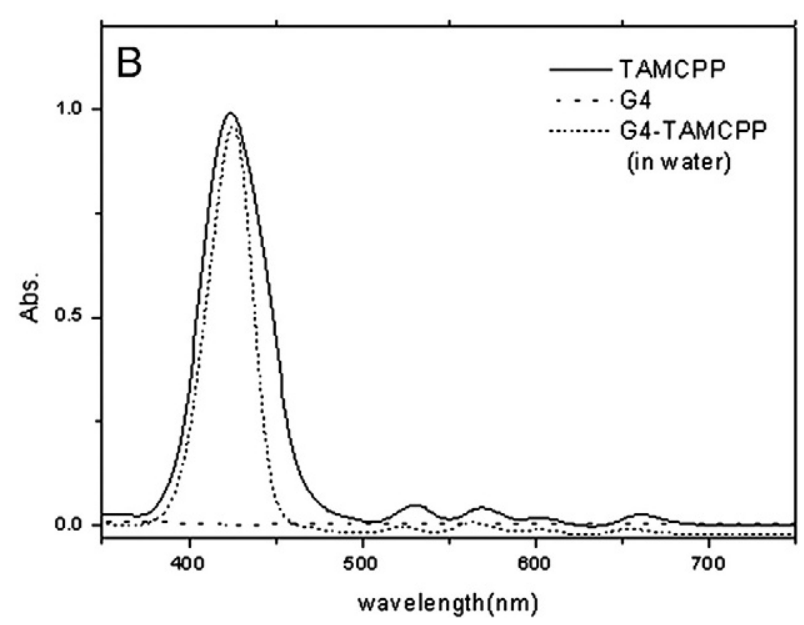

Fig. 1. TLC analysis (A) and absorption spectrum (B) of TAMCPP, PAMAM dendrimer and G4-TAMCPP conjugate. (A) Lane 1, PAMAM dendrimer; lane 2, TAMCPP; lane 3, G4TAMCPP; lane 4, G4+TAMCPP mixture.

photosensitizers at different concentration for $24 \mathrm{~h}$. After washing, cells were incubated with $1 \%$ SDS for $24 \mathrm{~h}$ at $37^{\circ} \mathrm{C}$. Cell lysates were collected and measured using a Cary Eclipse fluorescence spectrophotometer (Varian Inc., Palo Alto, CA, USA). Data were obtained from at least 3 independent experiments.

\subsection{Treatment of TAMCPP and G4-TAMCPP by photodynamic therapy (PDT)}

Cells were seeded into 96-well plates at a density of 3000 cells per well and cultured for $24 \mathrm{~h}$. To determine the cytotoxicity of the TAMCPP and G4-TAMCPP conjugates, cells were incubated in medium containing different concentrations of TAMCPP or G4-TAMCPP conjugates for $24 \mathrm{~h}$, washed, and then subjected to cell toxicity assays as described below.

To determine the cytotoxicity of TAMCPP-PDT, the cells were incubated with TAMCPP or G4-TAMCPP in 5-fold serial dilutions from $50 \mu \mathrm{M}$ to $0.4 \mu \mathrm{M}$ for $24 \mathrm{~h}$ at $37{ }^{\circ} \mathrm{C}$, washed, immediately exposed to different doses of light (1.4 or $2.8 \mathrm{~J} / \mathrm{cm}^{2}$ ), and then evaluated in cell toxicity assays. After the addition of TAMCPP or G4-TAMCPP, all the procedures were carried out in subdued light. The light source for activating TAMCPP was supplied by PCI Biotech (LumiSource ${ }^{\circledR}$, Oslo, Norway) and consisted of a bank of 4 fluorescent tubes (Osram 18W/67) emitting in the spectral region of $390-450 \mathrm{~nm}$ with the highest intensity at about $420 \mathrm{~nm}$. The total fluence rate was $7 \mathrm{~mW} / \mathrm{cm}^{2}$.

\subsection{Intracellular localization of the TAMCPP and G4-TAMCPP conjugates}

Cells were plated on glass coverslips in $35-\mathrm{mm}$ dishes the day before the experiment. After growing overnight, cells were exposed to TAMCPP and G4-TAMCPP compounds at $4 \mu \mathrm{M}$ for $24 \mathrm{~h}$. Cells were washed with PBS and examined directly or after co-staining with 100 nM LysoTracker Green (DND-26, Molecular Probes Inc, Leiden, The Netherlands) for $45 \mathrm{~min}$ at $37^{\circ} \mathrm{C}$, and then examined with a confocal microscope $\left(\lambda_{\mathrm{ex}}=488 \mathrm{~nm}\right.$; SP5 Spectral Confocal System; Leica, Nussloch, Germany). Fluorescence of the organelle probes was detected with an emission filter of $500-520 \mathrm{~nm}$, and porphyrins fluorescence was detected with an emission filter of 640-660 nm.

\subsection{Cytotoxicity assay}

Twenty-four hours after treatments, cytotoxicity was determined by using the MTT assay [23] and the results were read using a scanning multiwell ELISA reader (Microplate Autoreader EL311, Bio-Tek Instruments Inc., Winooski, VT, USA).

\subsection{Agarose gel electrophoresis studies}

The gel retardation assay was performed as follows. Polymer solutions were added to the enhanced green fluorescent protein (EGFP) plasmid DNA (pDNA, $4.7 \mathrm{~kb}$, Becton Dickinson, CA) solutions with the same volume at various [G4-TAMCPP]/[pDNA] charge ratios, shortly vortexed and incubated at room temperature overnight before use. The formation of complexes was analyzed by electrophoresis in $0.8 \%$ agarose gel and stained by ethidium bromide (EtBr) [10].

\subsection{Photochemical transfection}

For the preparation of dendrimer-plasmid DNA polyplexes, the EGFP plasmid DNA ( $2 \mu \mathrm{g}$ ) dissolved in $20 \mathrm{mM}$ Tris- $\mathrm{HCl}$ buffer was mixed with the G4-TAMCPP conjugates dissolved in distilled water. The mixed solution was added to serum-free DMEM to a final volume of $100 \mu \mathrm{L}$, and then incubated for $20 \mathrm{~min}$ at room temperature to allow formation of a polyplex with a given ratio of G4-TAMCPP primary amino groups to DNA phosphate (N/P ratio) [10]. The HeLa cells were seeded in 35-mm petri dishes the day before transfection. Cells were washed with PBS and then covered with fresh DMEM $(1.5 \mathrm{~mL})$. After 20-minute incubation, $0.4 \mathrm{~mL}$ of fresh DMEM was added to the mixed polyplex solution, and the polyplex solution was added gently to the cells and incubated at $37{ }^{\circ} \mathrm{C}$ for $24 \mathrm{~h}$. Cells were rinsed with PBS, covered with $1 \mathrm{~mL}$ of PBS, irradiated for 200 or $400 \mathrm{~s}$ [LumiSource®

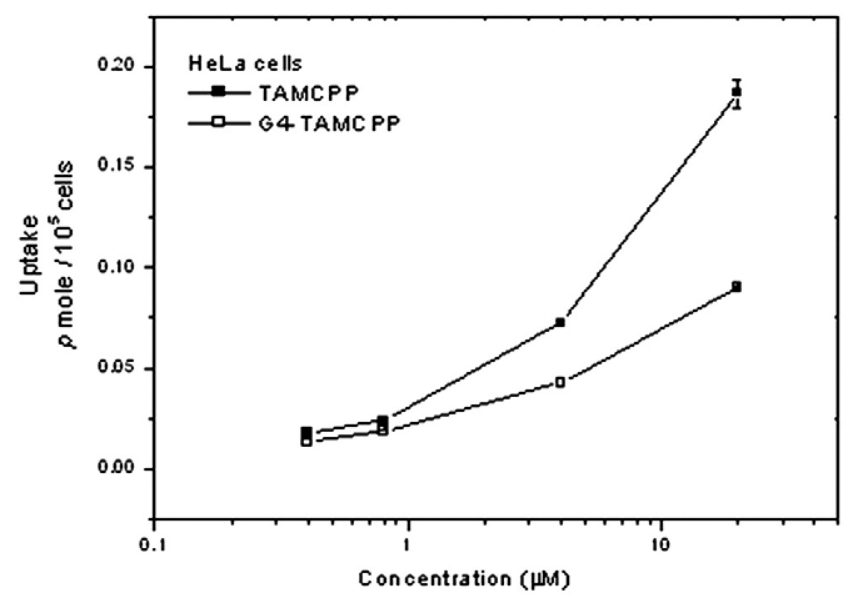

Fig. 2. Dose-dependent cellular uptake after $24 \mathrm{~h}$ incubation in HeLa cells. $(n=3)$. 


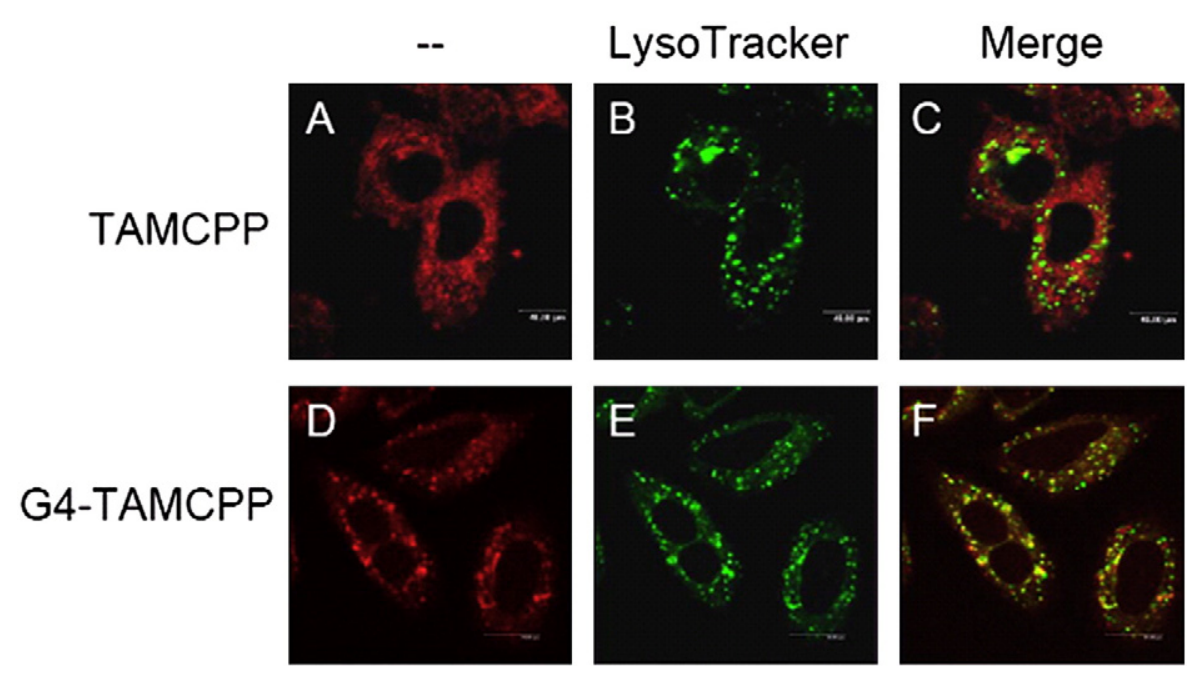

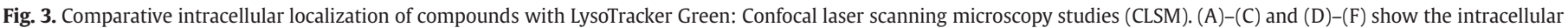
localization of TAMCPP and G4-TAMCPP, respectively.

(peak wavelength $435 \mathrm{~nm}, 7 \mathrm{~mW} / \mathrm{cm}^{2}$ )], and incubated at $37{ }^{\circ} \mathrm{C}$ for another $48 \mathrm{~h}$. The qualitative analysis of EGFP expression upon transfection in HeLa cells was evaluated by flow cytometry (FACS caliber, Becton Dickinson, USA) equipped with an argon 488 laser [24] and fluorescence microscopy (Zeiss, Axiophot 2 Microscope, Germany).

\subsection{Stability assay of lysosomal membrane}

HeLa cells were grown on glass slides in 35-mm petri dishes, incubated for $24 \mathrm{~h}$ with TAMCPP or G4-TAMCPP at $4 \mu \mathrm{M}$ and then irradiated with LumiSource ${ }^{\circledR}$ for $200 \mathrm{~s}$ or $400 \mathrm{~s}$. One hour after irradiation, cultures were incubated with $500 \mathrm{nM}$ acridine orange (AO) for 15 min before being sequentially imaged using filter cubes that captured red ( $\mathrm{AO}$ in an acidic $\mathrm{pH}$ environment) and green fluorescence ( $\mathrm{AO}$ in a neutral $\mathrm{pH}$ environment). $\mathrm{AO}$ was a metachromatic fluorophore and a lysosomotropic base $(p K a=10.3)$, which became charged $\left(\mathrm{AOH}^{+}\right)$and retained by proton trapping within acidic compartments, mainly secondary lysosomes ( $\mathrm{pH} 4.5-5.5$ ). When normal cells were excited by blue light, highly concentrated lysosomal AO emitted an intense red fluorescence, while nuclei and cytosol showed weak diffuse green fluorescence $[25,26]$. In this experiment, digital images were captured with fluorescence microscope system (Zeiss, Axiophot 2 Microscope, Germany).

\section{Results}

\subsection{Characterization of G4-TAMCPP conjugate}

The photosensitizer TAMCPP was conjugated to the surface amine of the PAMAM dendrimer, and then was dialyzed. Conjugation was confirmed by measuring the fluorescence intensity and by TLC analysis and infrared spectrometer [9,10]. As shown in Fig. 1AA, G4TAMCPP conjugate remained almost immobile at the origin, while unreacted TAMCPP migrated close to the solvent front, as observed under long wavelength UV light. The infrared spectra also showed that the peaks of $\mathrm{OH}$ and $\mathrm{COO}^{-}$stretch of TAMCPP observed at $3397 \mathrm{~cm}^{-1}$ and $1672 \mathrm{~cm}^{-1}$ disappeared after PAMAM dendrimer conjugation (G4TAMCPP), thereby demonstrating the formation of the covalent bond between PAMAM dendrimers and TAMCPP molecules (see Supplementary data).

The absorption spectra of TAMCPP and G4-TAMCPP in DMSO are shown in Fig. 1B. After conjugating to the PAMAM dendrimer, the absorption peak of TAMCPP did not significantly shift, with a typical high-intensity soret band near $420 \mathrm{~nm}$ ( $\log \varepsilon \sim 5.62$ for TAMCPP) and a low intensity $Q$ band between 500 and $700 \mathrm{~nm}$. The conjugated porphyrin to the PAMAM dendrimer was also determined by UV-VIS spectrophotometer using $420 \mathrm{~nm}$ for porphyrin absorbance. Our result showed that four porphyrins were covalently attached to one G4 PAMAM dendrimer.

\subsection{Cell uptake and intracellular localization of TAMCPP and G4-TAMCPP}

Fig. 2 shows the uptake of TAMCPP and G4-TAMCPP by HeLa cells as a function of the concentration of the photosensitizers. At the concentration of $0.4 \mu \mathrm{M}$ or $0.8 \mu \mathrm{M}$, there was no significant difference in the cellular uptake of TAMCPP and G4-TAMCPP conjugates. Cellular uptake of TAMCPP was significantly higher than that of G4-TAMCPP at $4 \mu \mathrm{M}$ or $20 \mu \mathrm{M}$ (2-fold), probably due to the hydrophobic property of TAMCPP, which caused more permeation and accumulation in the cells [27]. Differently from the TAMCPP, which can diffuse across the cell membrane and enter cells, G4-TAMCPP might be taken by cells via endocytosis [5,6]. After cellular endocytosis, G4-TAMCPP was entrapped presumably in the endosomes/lysosomes, which can be confirmed by confocal microscopy. As shown in Fig. 3, the fluorescence pattern of G4-TAMCPP (red) was almost co-localized with LysoTracker (green) in HeLa cells. However, TAMCPP was co-localized with

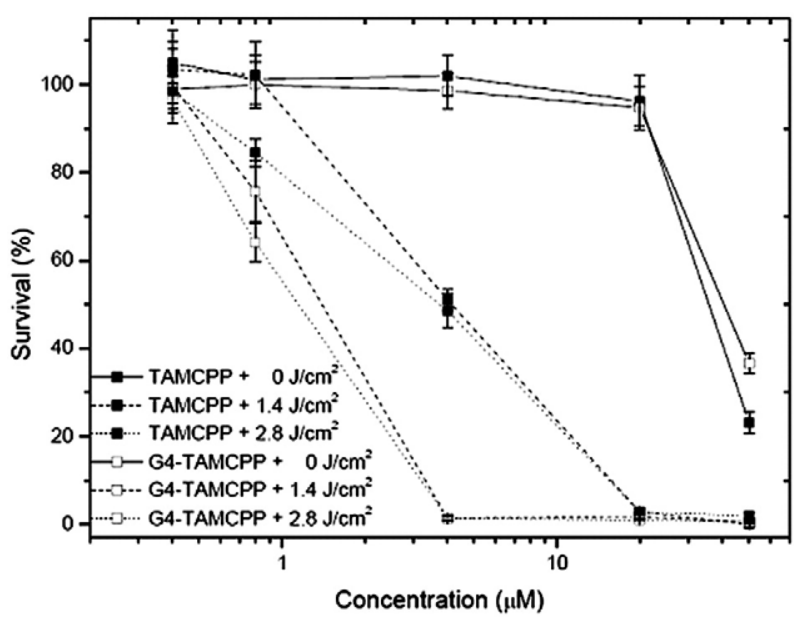

Fig. 4. Cytotoxicity and photocytoxicity of TAMCPP and G4-TAMCPP conjugate. Cells were incubated for $24 \mathrm{~h}$ at $37{ }^{\circ} \mathrm{C}$ w/o or w/irradiation $(n=4)$. 

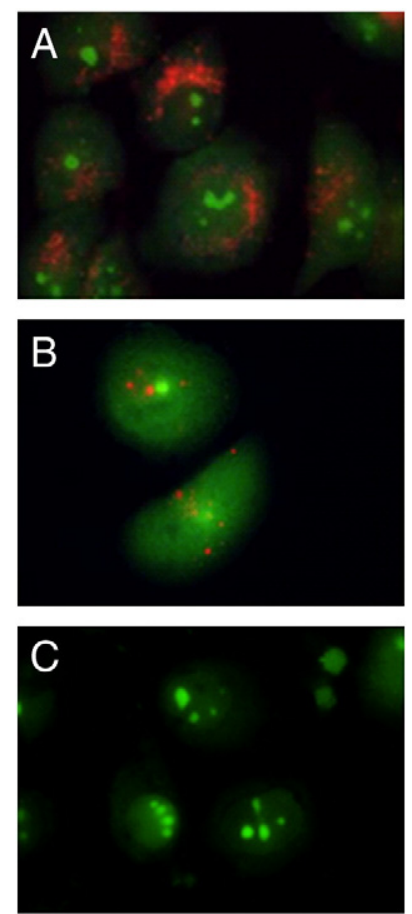

Fig. 5. Estimation of lysosome disruption capability of $4 \mu \mathrm{M}$ G4-TAMCPP conjugate. After $24 \mathrm{~h}$ incubation of G4-TAMCPP, cells were irradiated with LumiSource ${ }^{\circledR}$ for $200 \mathrm{~s}$ (B) and $400 \mathrm{~s}$ (C). After $1 \mathrm{~h}$, the cells were stained with acridine orange for $15 \mathrm{~min}$ and measured by fluorescence microscope. (A) is the control without irradiation.

lysosomes, and was considered to accumulate not only in lysosomes but also in other organelles in the cytosol.

\subsection{Cytotoxicity and phototoxicity}

The dose-dependent cytotoxicity and phototoxicity of the photosensitizing agents in HeLa cells is shown in Fig. 4. Without irradiation, more than $94 \%$ of the cells survived after $20 \mu \mathrm{M}$ TAMCPP or G4TAMCPP incubation. However, TAMCPP and G4-TAMCPP induced cell toxicity at $50 \mu \mathrm{M}$ ( $23 \%$ and $36 \%$ cell survival, respectively). For photodynamic therapy, there was no significant toxicity of TAMCPP or G4-TAMCPP at $0.4 \mu \mathrm{M}$ with 1.4 or $2.8 \mathrm{~J} / \mathrm{cm}^{2}$ light dose. Photoinduced cytotoxicity was observed with G4-TAMCPP at $4 \mu \mathrm{M}$ (more than $98 \%$ cell death), whereas $51 \%$ the cells survived TAMCPP incubation with $1.4 \mathrm{~J} / \mathrm{cm}^{2}$ light dose. The phototoxicity of TAMCPP was greater with a higher light dose. At $0.8 \mu \mathrm{M}, 16 \%$ of the cells were killed by TAMCPP with the light dose of $2.8 \mathrm{~J} / \mathrm{cm}^{2}$, but most of the cells were still viable with the light dose of $1.4 \mathrm{~J} / \mathrm{cm}^{2}$. Similarly to TAMCPP, cell toxicity induced by G4-TAMCPP increased from $25 \%$ to $36 \%$ with an increasing light dose. Obviously, $0.4 \mu \mathrm{M}$ G4-TAMCPP with 1.4 or $2.8 \mathrm{~J} / \mathrm{cm}^{2}$ light dose was non-toxic (Fig. 4), and was further evaluated for photochemical transfection.

\subsection{Photochemical disruption of the endosomal/lysosomal membrane}

To assess whether G4-TAMCPP conjugates could disrupt the endosomal/lysosomal membrane and could help gene escape from the lysosomes after irradiation, AO was utilized as a marker to trace the integrity of the endosomes/lysosomes. As shown in Fig. 5A, acidic organelles (endosomes/lysosomes) of cells treated with G4-TAMCPP could be stained by AO without light (red spots). After irradiation, the red spots decreased with a $1.4 \mathrm{~J} / \mathrm{cm}^{2}$ light dose (Fig. 5B) and almost disappeared with a $2.8 \mathrm{~J} / \mathrm{cm}^{2}$ light dose (Fig. 5C). These results demonstrated that the endocytozed G4-TAMCPP, which accumulated in the endosomes/lysosomes, could destabilize the endosomal/

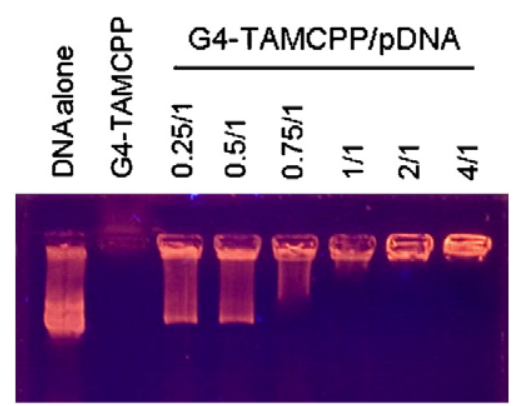

Fig. 6. Gel retardation assay for G4-TAMCPP. $2 \mu \mathrm{g}$ pDNA was mixed with G4-TAMCPP conjugate according to the present charge ratios and then analyzed by electrophoresis with $\mathrm{EtBr}$ staining.

lysosomal membranes and cause a rapid loss of AO staining of acidic organelles after irradiation.

\subsection{Evaluation of complex formation with $p D N A$}

One prerequisite for the usefulness of polycationic gene carriers is to efficiently form polymer/DNA complexes, a process that can be confirmed by retardation of DNA mobility in agarose gel electrophoresis. PAMAM dendrimers could efficiently form complexes with pDNA through electrostatic interaction between the protonated primary amines of the dendrimers and negatively charged phosphate groups on the pDNA. It was found that the G4-TAMCPP conjugate was able to retard DNA mobility in gels; however, retardation was complete only when an equivalence or an excess of dendrimer amino groups was present (Fig. 6).

\subsection{Photochemical transfection of G4-TAMCPP conjugates}

To evaluate the transfection efficiency of the dendrimer/pDNA polyplex with or without irradiation at non-toxic conditions, G4TAMCPP at $0.4 \mu \mathrm{M}$ was used with the light dose of 1.4 or $2.8 \mathrm{~J} / \mathrm{cm}^{2}$ light dose. Fig. 7 shows the cell viability and photochemical transfection efficiency of the dendrimer/pDNA polyplex in HeLa cells. Without irradiation, less than $3.5 \%$ of the cells were transfected by G4-TAMCPP conjugate, similarly to that observed with the G4 PAMAM dendrimer in a previous report [7]. After $24 \mathrm{~h}$ incubation, G4-TAMCPP/pDNA polyplex were almost co-localized with LysoTracker (green) (data not shown). Surprising, G4-TAMCPP conjugate resulted in the transfection of $12.6 \%$ and $26.7 \%$ of the cells using a 1.4 and $2.8 \mathrm{~J} / \mathrm{cm}^{2}$ of light dose,

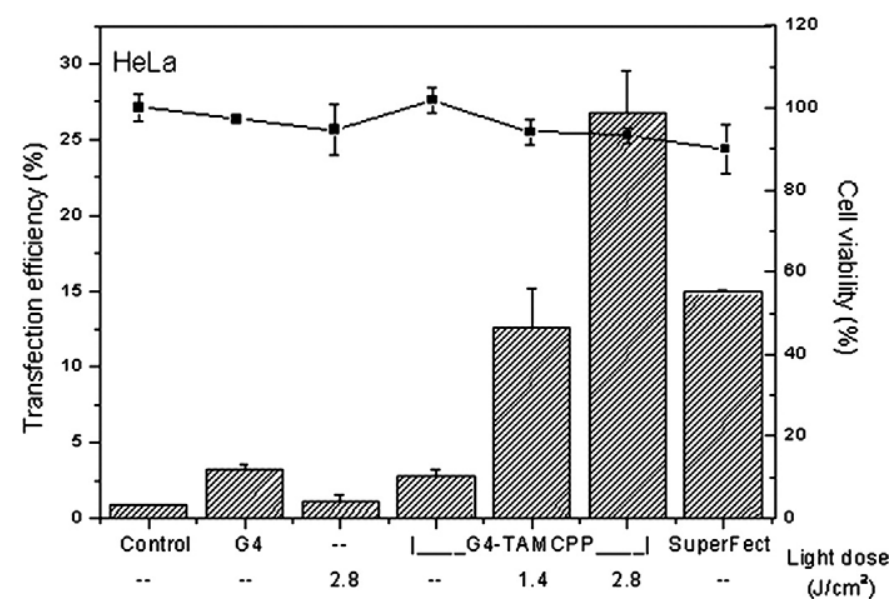

Fig. 7. Cell viability (line) and light-triggered transfection efficiency (column) of polyplex (dendrimer: $0.4 \mu \mathrm{M}$ ) with different light doses $(n=3)$. 
respectively. Compared with G4-TAMCPP conjugate alone, the transfection efficiency was improved more than 9.5-fold with a $2.8 \mathrm{~J} / \mathrm{cm}^{2}$ light dose of irradiation, which represented almost twice the efficiency of the SuperFect commercial kit. It is of note that the photochemical transfection of G4-TAMCPP/pDNA polyplex with a $2.8 \mathrm{~J} / \mathrm{cm}^{2}$ light dose left more than $93 \%$ of the cells viable (Fig. 7). Therefore, irradiation can be a "switch" to control gene transfection in this system.

\section{Discussion}

There has been a substantial interest in the use of polycationic materials to deliver plasmid DNA, antisense oligonucleotides, and small interfering RNA $[7,28,29]$. While high transfection efficiency has been reported in a number of cases, toxicity of these non-viral vectors is an important problem [30]. Besides, controllable gene transfection is another interesting technique to be developed for biomedical application. In this study, a non-toxic, controllable gene delivery system was developed using PAMAM dendrimer-porphyrin conjugates with suitable light irradiation.

PAMAM dendrimer is a well-known cationic polymer containing amino groups with weak base properties that can possess the capacity to buffer $\mathrm{pH}$ in the endosomes/lysosomes. Recently, Sonawane et al. demonstrated that the average $\mathrm{pH}$ in endosomes/lysosomes containing G6 fractured PAMAM dendrimer decreased slowly from 7.2 to 5.9 over $60 \mathrm{~min}$ and increased to 6.5 at $75 \mathrm{~min}$ in Chinese hamster ovaryK1 cells and the buffering capacity of PAMAM dendrimer was attributed to proton sponge effects [31]. Results from our lab showed that the endosomal/lysosomal pH was buffered by G4 PAMAM dendrimer and was remained at 6.8 over $12 \mathrm{~h}$ in Ca9-22 cells [32]. Even after $48 \mathrm{~h}$ incubation, G4 PAMAM dendrimer was still entrapped into endosomes/lysosomes that may cause the inefficient transfection $[7,31,33,34]$. Thus, the capacity of $\mathrm{pH}$ buffering of cationic polymer in endosome/lysosomes is not the only reason to achieve higher transfection efficiency. Recently, surface conjugation to low generation of PAMAM dendrimer surface by hydrophobic moieties may be a strategy to improve the inefficient transfection property. Yoo et al. reported that the delivery of antisense oligonucleotides can be enhanced by fluorophore-conjugated PAMAM dendrimers because the relative hydrophobicity of fluor molecules can enhance endosomes/lysosomes disruption although this mechanism remains unclear [9]. Similarly, the transfection efficiency of PAMAM dendrimer also can be improved by hydrophobic amino acid conjugation [10]. In this study, hydrophobic porphyrin porphyrin-conjugated G4 PAMAM dendrimer shows an unapparent improvement in AO-stained acidic organelles loss or gene transfection, which can be further enhanced conspicuously by irradiation. This controllable gene delivery was supposed as PCI effects.

$\mathrm{PCI}$ is an interesting strategy to improve the cytosolic release of macromolecules from the endosomes/lysosomes in a light-inducible manner. In the previous studies, asymmetric photosensitizers, such as AlPcS2a and TPPS2a, were effective in PCI-induced delivery because these photosensitizers prefer plasma membrane localization via hydrophobic-hydrophobic interactions [35,36]. However, these photosensitizers could re-localize to other organelles, thereby increasing phototoxicity, an important issue that needed to be considered for further PCI applications [36-38]. Besides, most gene vectors and photosensitizers need to be administrated separately in PCI-enhanced gene delivery systems because of limitations of the carrier capability of these photosensitizers. Therefore, the development of a potential gene carrier with low phototoxic and controllable properties for effective PCI-mediated gene delivery is a challenge in biomaterial sciences. The synthesized G4-TAMCPP conjugate in this study processes the potential to be a controllable non-viral vector. Our results show that the G4-TAMCPP conjugate was uptaken by HeLa cells and accumulated in endosomes/lysosomes compartments via the endocytotic pathway. The punctate pattern exhibited by AO-stained acidic organelles was significantly reduced after irradiation that may due to the destabilization of endosomal/lysosomal membranes (Fig. 5). Not only nanoparticle-based photosensitizers, the lysosomal targeting photosensitizer, like $\mathrm{N}$-aspartyl chlorin e6 (NPe6), exhibited the similar AO-staining pattern after irradiation [26]. Therefore, this photochemical reaction may release the entrapped pDNA from the endosome/lysosome to the cystosol and activate the gene transfection process.

Of note, almost non-toxic transfection was achieved using $0.4 \mu \mathrm{M}$ G4-TAMCPP plus irradiation, representing an important achievement (Fig. 7). With non-toxic light doses, G4-TAMCPP still reveals effective at expressing EGFP in HeLa cells. G4-TAMCPP plus a $2.8 \mathrm{~J} / \mathrm{cm}^{2}$ light dose had greater transfection efficiency than the other nanocarriers as well as SuperFect-pDNA complexes or other treatments [39-41]. The phototoxicity is lower than with other membrane-accumulated photosensitizers, such as AIPCS2a or TPPS2a, which may due to the localization of G4-TAMCPP in the endosomes/lysosomes. Moreover, because the electrostatic forces play a dominant role in the affinity and dissociation of DNA to/from cationic polymer, the dissociation of polyplex can occur by the intracellular polyion [42]. The PAMAM dendrimer might also be degraded after being taken up by the cells, which would result in a gradual reduction in positive charge density and a consequential change in the electrostatic interactions between DNA and PAMAM, and facilitate the dissociation of DNA from the polyplex [43]. Consequently, G4-TAMCPP may be dissociated from pDNA in endosomes/lysosomes and translocated to the endosomal/ lysosomal membranes because of the relatively hydrophobicity of the porphyrin on the PAMAM dendrimer surface, which may also prevent photochemical damage to the pDNA and cause more efficient transfection [37]. Therefore, porphyrin-conjugated PAMAM dendrimer plus suited irradiation is an almost non-toxic and light-inducible effective gene delivery strategy. Based on these results, in vivo gene transfection studies by porphyrin-conjugated PAMAM dendrimer with light irradiation should be encouraged.

\section{Conclusion}

In this study, we have shown that PAMAM dendrimers conjugated with porphyrin is a potential gene carrier. Effective gene transfection can be controlled by light-induced endosomes/lysosomes disruption, which may cause the entrapped gene to be released into the cytosol. Moreover, this procedure seems to be safe (low toxicity) and therefore is a satisfactory alternative photoinducible non-viral gene delivery strategy for the future in vivo development of PCI-medicated gene delivery system.

\section{Acknowledgement}

This research was supported by grants from National Science Council of the Republic of China (NSC 95-2221-E-002-025 and NSC 962113-M-005-015).

\section{Appendix A. Supplementary data}

Supplementary data associated with this article can be found, in the online version, at doi:10.1016/j.jconrel.2008.03.024.

\section{References}

[1] Q. Liu, D.A. Muruve, Molecular basis of the inflammatory response to adenovirus vectors, Gene Ther. 10 (11) (2003) 935-940.

[2] J.Y. Sun, V. Anand-Jawa, S. Chatterjee, K.K. Wong, Immune responses to adenoassociated virus and its recombinant vectors, Gene Ther. 10 (11) (2003) 964-976.

[3] L. De Laporte, J.C. Rea, L.D. Shea, Design of modular non-viral gene therapy vectors, Biomaterials 27 (7) (2006) 947-954.

[4] S. Han, R.I. Mahato, Y.K. Sung, S.W. Kim, Development of biomaterials for gene therapy, Mol. Ther. 2 (4) (2000) 302-317. 
[5] C.C. Lee, J.A. MacKay, J.M.J. Frechet, F.C. Szoka, Designing dendrimers for biological applications, Nat. Biotechnol. 23 (12) (2005) 1517-1526.

[6] R. Esfand, D.A. Tomalia, Poly(amidoamine) (PAMAM) dendrimers: from biomimicry to drug delivery and biomedical applications, Drug Discov. Today 6 (8) (2001) 427-436.

[7] J.F. KukowskaLatallo, A.U. Bielinska, J. Johnson, R. Spindler, D.A. Tomalia, J.R. Baker, Efficient transfer of genetic material into mammalian cells using Starburst polyamidoamine dendrimers, Proc. Natl. Acad. Sci. U. S. A. 93 (10) (1996) 4897-4902.

[8] J.C. Roberts, M.K. Bhalgat, R.T. Zera, Preliminary biological evaluation of polyamidoamine (PAMAM) Starburst (TM) dendrimers, J. Biomed. Mater. Res. 30 (1) (1996) 53-65.

[9] H. Yoo, R.L. Juliano, Enhanced delivery of antisense oligonucleotides with fluorophore-conjugated PAMAM dendrimers, Nucleic Acids Res. 28 (21) (2000) 4225-4231.

[10] K. Kono, H. Akiyama, T. Takahashi, T. Takagishi, A. Harada, Transfection activity of polyamidoamine dendrimers having hydrophobic amino acid residues in the periphery, Bioconjug. Chem. 16 (1) (2005) 208-214.

[11] T.J. Dougherty, C.J. Gomer, B.W. Henderson, G. Jori, D. Kessel, M. Korbelik, J. Moan, Q. Peng, Photodynamic therapy, J. Natl. Cancer Inst. 90 (12) (1998) 889-905.

[12] K. Berg, P.K. Selbo, L. Prasmickaite, T.E. Tjelle, K. Sandvig, D. Moan, G. Gaudernack, O. Fodstad, S. Kjolsrud, H. Anholt, G.H. Rodal, S.K. Rodal, A. Hogset, Photochemical internalization: a novel technology for delivery of macromolecules into cytosol, Cancer Res. 59 (6) (1999) 1180-1183.

[13] K. Berg, A. Dietze, O. Kaalhus, A. Hogset, Site-specific drug delivery by photochemical internalization enhances the antitumor effect of bleomycin, Clin. Cancer Res. 11 (23) (2005) 8476-8485.

[14] A. Ndoye, J.L. Merlin, A. Leroux, G. Dolivet, P. Erbacher, J.P. Behr, K. Berg, F. Guillemin, Enhanced gene transfer and cell death following P53 gene transfer using photochemical internatisation internalisation of glucosylated PEI-DNA complexes, J. Gene Med. 6 (8) (2004) 884-894.

[15] L. Prasmickaite, L. Cekaite, M. Hellum, E. Hovig, A. Hogset, K. Berg, Transcriptome changes in a colon adenocarcinoma cell line in response to photochemical treatment as used in photochemical internalisation (PCI), FEBS Lett. 580 (24)(2006) 5739-5746.

[16] A. Weyergang, P.K. Selbo, K. Berg, Photochemically stimulated drug delivery increases the cytotoxicity and specificity of EGF-saporin, J. Control. Release 111 (1-2) (2006) 165-173.

[17] L. Prasmickaite, A. Hogset, P.K. Selbo, B.O. Engesaeter, M. Hellum, K. Berg, Photochemical disruption of endocytic vesicles before delivery of drugs: a new strategy for cancer therapy, Br. J. Cancer 86 (4) (2002) 652-657.

[18] M. Folini, K. Berg, E. Millo, R. Villa, L. Prasmickaite, M.G. Daidone, U. Benatti, N. Zaffaroni, Photochemical internalization of a peptide nucleic acid targeting the catalytic subunit of human telomerase, Cancer Res. 63 (13) (2003) 3490-3494.

[19] P.S. Lai, P.J. Lou, C.L. Peng, C.L. Pai, W.N. Yen, M.Y. Huang, T.H. Young, M.J. Shieh, Doxorubicin delivery by polyamidoamine dendrimer conjugation and photochemical internalization for cancer therapy, J. Control. Release 122 (1) (2007) 39-46.

[20] P.J. Lou, P.S. Lai, M.J. Shieh, A.J. MacRobert, K. Berg, S.G. Bown, Reversal of doxorubicin resistance in breast cancer cells by photochemical internalization, Int. J. Cancer 119 (11) (2006) 2692-2698.

[21] A.D. Adler, F.R. Longo, J.D. Finarell, J. Goldmach, J. Assour, L. Korsakof, A simplified synthesis for meso-tetraphenylporphin, J. Org. Chem. 32 (2) (1967) 476-478

[22] A. Leo, C. Hansch, D. Elkins, Partition coefficients and their uses, Chem. Rev. 71 (6) (1971) 525-616.

[23] T. Mosmann, Rapid colorimetric assay for cellular growth and survival applicarion application to proliferation and cyto-toxicity assays, J. Immunol. Methods 65 (1-2) (1983) 55-63.

[24] S. Kommareddy, M. Amiji, Preparation and evaluation of thiol-modified gelatin nanoparticles for intracellular DNA delivery in response to glutathione, Bioconjug. Chem. 16 (6) (2005) 1423-1432.
[25] T. Kurz, B. Gustafsson, U.T. Brunk, Intralysosomal iron chelation protects against oxidative stress-induced cellular damage, FEBS J. 273 (13) (2006) 3106-3117.

[26] J.A. Caruso, P.A. Mathieu, J.J. Reiners, Sphingomyelins suppress the targeted disruption of lysosomes/endosomes by the photosensitizer NPe6 during photodynamic therapy, Biochem. J. 392 (2005) 325-334.

[27] R.K. Pandey, A.B. Sumlin, S. Constantine, M. Aoudia, W.R. Potter, D.A. Bellnier, B.W Henderson, M.A. Rodgers, K.M. Smith, T.J. Dougherty, Alkyl ether analogs of chlorophyll-a derivatives .1. Synthesis, photophysical properties and photodynamic efficacy, Photochem. Photobiol. 64 (1) (1996) 194-204.

[28] J.R. Kovacs, Y. Zheng, H.M. Shen, W.S. Meng, Polymeric microspheres as stabilizing anchors for oligonucleotide delivery to dendritic cells, Biomaterials 26 (33) (2005) 6754-6761.

[29] J.H. Jeong, L.V. Christensen, J.W. Yockman, Z.Y. Zhong, J.F.J. Engbersen, W.J. Kim, J. Feijen, S.W. Kim, Reducible poly(amido ethylenimine) directed to enhance RNA interference, Biomaterials 28 (10) (2007) 1912-1917.

[30] T.I. Kim, J.U. Baek, C.Z. Bai, J.S. Park, Arginine-conjugated polypropylenimine dendrimer as a non-toxic and efficient gene delivery carrier, Biomaterials 28 (11) (2007) 2061-2067.

[31] N.D. Sonawane, F.C. Szoka, A.S. Verkman, Chloride accumulation and swelling in endosomes enhances DNA transfer by polyamine-DNA polyplexes, J. Biol. Chem. 278 (45) (2003) 44826-44831.

[32] P.S. Lai, C.L. Pai, C.L. Peng, M.J. Shieh, K. Berg P.J. Lou, Enhanced cytotoxicity of saporin by polyamidoamine dendrimer conjugation and photochemical internalization. J. Biomed. Mater. Res. Part A (in press).

[33] C. Plank, K. Mechtler, F.C. Szoka, E. Wagner, Activation of the complement system by synthetic DNA complexes: a potential barrier for intravenous gene delivery, Human Gene Ther. 7 (12) (1996) 1437-1446.

[34] M.X. Tang, C.T. Redemann, F.C. Szoka, In vitro gene delivery by degraded polyamidoamine dendrimers, Bioconjug. Chem. 7 (6) (1996) 703-714.

[35] I.J. MacDonald, T.J. Dougherty, Basic principles of photodynamic therapy, J. Porphyr. Phthalocyanines 5 (2) (2001) 105-129.

[36] J. Moan, K. Berg, H. Anholt, K. Madslien, Sulfonated aluminum phthalocyanines as sensitizers for photochemotherapy - effects of small light doses on localization, dye fluorescence and photosensitivity in V79 cells, Int. J. Cancer 58 (6) (1994) $865-870$.

[37] N. Nishiyama, A. Iriyama, W.D. Jang K. Miyata, K. Itaka, Y. Inoue, H. Takahashi, Y. Yanagi, Y. Tamaki, H. Koyama, K. Kataoka, Light-induced gene transfer from packaged DNA enveloped in a dendrimeric photosensitizer, Nat. Matters 4(12)(2005) 934-941.

[38] G.H. Rodal, S.K. Rodal, J. Moan, K. Berg, Liposome-bound Zn(II)-phthalocyanine. Mechanisms for cellular uptake and photosensitization J. Photochem. Photobiol., B Biol. 45 (2-3) (1998) 150-159.

[39] M. Turk, S. Dincer, I.S. Yulug, E. Piskin, In vitro transfection of HeLa cells with temperature sensitive polycationic copolymers, J. Control. Release 96 (2) (2004) 325-340.

[40] L.B. Feril, R. Ogawa, K. Tachibana, T. Kondo, Optimized ultrasound-mediated gene transfection in cancer cells, Cancer Sci. 97 (10) (2006) 1111-1114.

[41] S.W. Kamau, P.O. Hassa, B. Steitz, A. Petri-Fink, H. Hofmann, M. Hofmann-Amtenbrink, B. von Rechenberg, M.O. Hottiger, Enhancement of the efficiency of non-viral gene delivery by application of pulsed magnetic field, Nucleic Acids Res. 34 (5) (2006) e40.

[42] S. Katayose, K. Kataoka, Water-soluble polyion complex associates of DNA and poly (ethylene glycol)-poly(L-lysine) block copolymer, Bioconjug. Chem. 8 (5) (1997) 702-707.

43] L.Z. Zhou, L. Gan, H.B L, X.L. Yang, Studies on the interactions between DNA and PAMAM with fluorescent probe $\left[\mathrm{Ru}(\mathrm{phen})_{2} \mathrm{dppz}\right]^{2+}, \mathrm{J}$. Pharm. Biomed. Anal. 43 (1) (2007) 330-334. 